\title{
Physico-Chemical Differences Between Particle- and Molecule-Derived Toxicity: Can We Make Inherently Safe Nanoparticles?
}

\author{
Ludwig K. Limbach, Robert N. Grass, and Wendelin J. Stark*
}

\begin{abstract}
The rapidly growing applications of nanotechnology require a detailed understanding of benefits and risks, particularly in toxicology. The present study reviews the physical and chemical differences between particles and molecules when interacting with living organisms. In contrast to classical chemicals, the mobility of nanoparticles is governed by agglomeration, a clustering process that changes the characteristic size of the nanomaterials during exposure, toxicity tests or in the environment. The current status of nanotoxicology highlights non-classical toxic interactions through catalytic processes inside living cells and the enhanced heavy metal transport into the cytosol through the 'Trojan horse mechanism'. The safety of nanoparticles in consumer goods is proposed to be rendered inherently safer by substituting the currently used persistent oxides through biodegradable materials.
\end{abstract}

Keywords: Ecotoxicology · Engineered nanoparticles · Nanotoxicology $\cdot$ Persistence $\cdot$ Risk

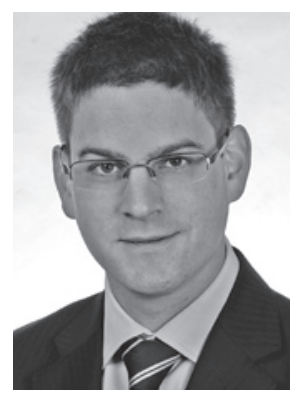

Wendelin Stark (1976) founded the Functional Materials Laboratory at ETH Zürich in 2004. His interdisciplinary research is situated at the interface between materials, chemistry and medicine. An applicationoriented focus has resulted in industrialscale production of Lotus-type non-adhesive polymers, self-sterilizing surfaces and two spin-off companies (Turbobeads and Nanograde). An injectable and resorbable bone cement is currently undergoing intense preclinical testing at the University Hospital in Zürich. Research in nanotoxicology routinely accompanies product development and assists a sustainable implementation of nanoparticles in industrial applications.

\section{Nanotechnology: Exponential Growth and Increasing Concerns}

For engineers (and most other people) seeing is believing: The discovery of the scanning tunneling microscope ${ }^{[1]}$ has allowed imaging at the nanometer range. The resulting engineering and science discipline 'nanotechnology' is only broadly defined because of its huge diversity. 'Nano', the metric label for $10^{-9}$, now serves as a thematic umbrella in nearly all scientific disciplines and has created enormous financial, medical, and social expectations, together with an exponential growth in scientific publishing activity. The associated risks of such a technology change, however, have only slowly shifted into the focus of scientists during the last few years. ${ }^{[2,3]}$ Most concerns have been related to nanoparticles, a sub-discipline of nanotechnology. Negative headlines mostly target nanoparticles and refer to their similarity to particulate air pollution ${ }^{[4]}$ which was found responsible for increased pulmonary and cardiovascular mortality in numerous epidemiological studies. ${ }^{[5]}$ There, most adverse health effects could be correlated to the fraction of ultrafine particles (UFP, $<100 \mathrm{~nm})^{[6]}$ but their characterization has challenged scientists for years. ${ }^{[7]}$ Regional changing composition and the small concentrations of UFP in air complicate mechanistic correlations of effects on health to specific chemical or physical properties of fine dust.

In contrast, nanotoxicology now investigates human exposure to mostly well- characterized nanoparticles using model animals or cell lines. ${ }^{[8-10]}$ The combination of this young discipline and epidemiological exposure data on ultrafine particles will, therefore, provide the key to the design of inherently safer nanomaterials before larger incidents might corrode the (currently) very positive perception of 'nano' to the general public.

Guidelines for the development of inherently safer nanoparticles are of imminent interest to industry and governmental agencies and should assist applicationoriented research. Early identification of critical materials helps to avoid costly latestage corrections and reduces the risk of large-scale incidents.

With the present article we would like to summarize briefly the current understanding of nanoparticle cell interactions and to identify key properties to assist regulatory efforts ${ }^{[11]}$ on the proactive design of inherently safer nanoparticles. At present a lack of base data (mechanism and individual material data) may stimulate governmental (over-) regulation of nanoparticles and, in turn, slow down most attractive technology developments. In order to guarantee long-term benefits from nanoparticle-related products, we therefore urgently need proactive material assessment methods, until more detailed toxicology data has become available. In order to develop such tools we may go back one step and look into the development of chemical regulatory systems. There, approval and use of specific substances is heavily regulated; 
governmental agencies take responsibility for consumers and give security to investors. This elaborate system has grown over decades. With engineered nanoparticles such a time delay would be unacceptable. A 'wait and see' strategy will result in a sequence of incidents and resulting corrections, with the associated loss of faith in the technology.

\section{Nanoparticles vs. Molecules or Microparticles: What is Physically Different?}

Chemicals are classified according to several key risk factors such as flammability, oxidizing power, damage to aquatic systems, human short- (acute) and longcal toxicological assay the tested material (i.e. a chemical or a mixture) has to be exposed to biological fluids, consisting mainly of water, salts and biomolecules. Both in vitro (cell-based) or in vivo (animal) tests inherently follow this route. Unfortunately, chemicals and nanoparticles behave completely differently in stationary fluids, which are typically used in in vitro assays: The physical driving force for the movement of molecules is diffusion. This rapid movement of molecules counteracts any concentration gradient and assists distribution of so-called 'soluble' molecule homogeneously into its corresponding phase (cytosol, blood, body fat, etc.). Microparticles and 'larger' objects have different driving forces withterm toxicity and many others. In a typi-
Scheme. Nanoparticles tend to form clusters (so called agglomerates). These fractal structures occur when attractive particle-particle forces overcome stabilization.

$$
\begin{gathered}
\circ \\
0 \\
0 \\
0 \\
0 \\
0 \\
0 \\
0
\end{gathered}
$$

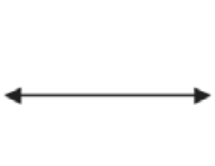

un-agglomerated

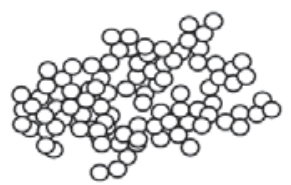

agglomerated in stagnant media and involve sedimentation or buoyancy. This is most obvious by mixing sand in water; after turning off the stirrer the sand will sediment to the ground. Within the size range of nanomaterials, however, a crossover takes place from diffusion-controlled movement to sedimentation-controlled movement (Fig. 1). In order to illustrate this, Fig. $1 \mathrm{com}-$ pares the size of different 'nanomaterials' to the size of chemicals and atoms.

When we talk about molecules or chemicals we typically characterize them by solubility, reactivity and their chemical nature (connectivity of the atoms, chirality). Scientists working with nanomaterials intuitively try to use the same concepts when assessing toxicology, medical applications or environmental spread of a nanomaterial. Unfortunately this is wrong, as an additional physical property often dominates particle behavior in the nanometer range. As particles in a medium (air, water, fat, etc.) are always in a metastable form, they can only be temporarily stabilized, typically by charge or steric repulsion. If particles stick together they form clusters or so-called agglomerates (Scheme). Ag-

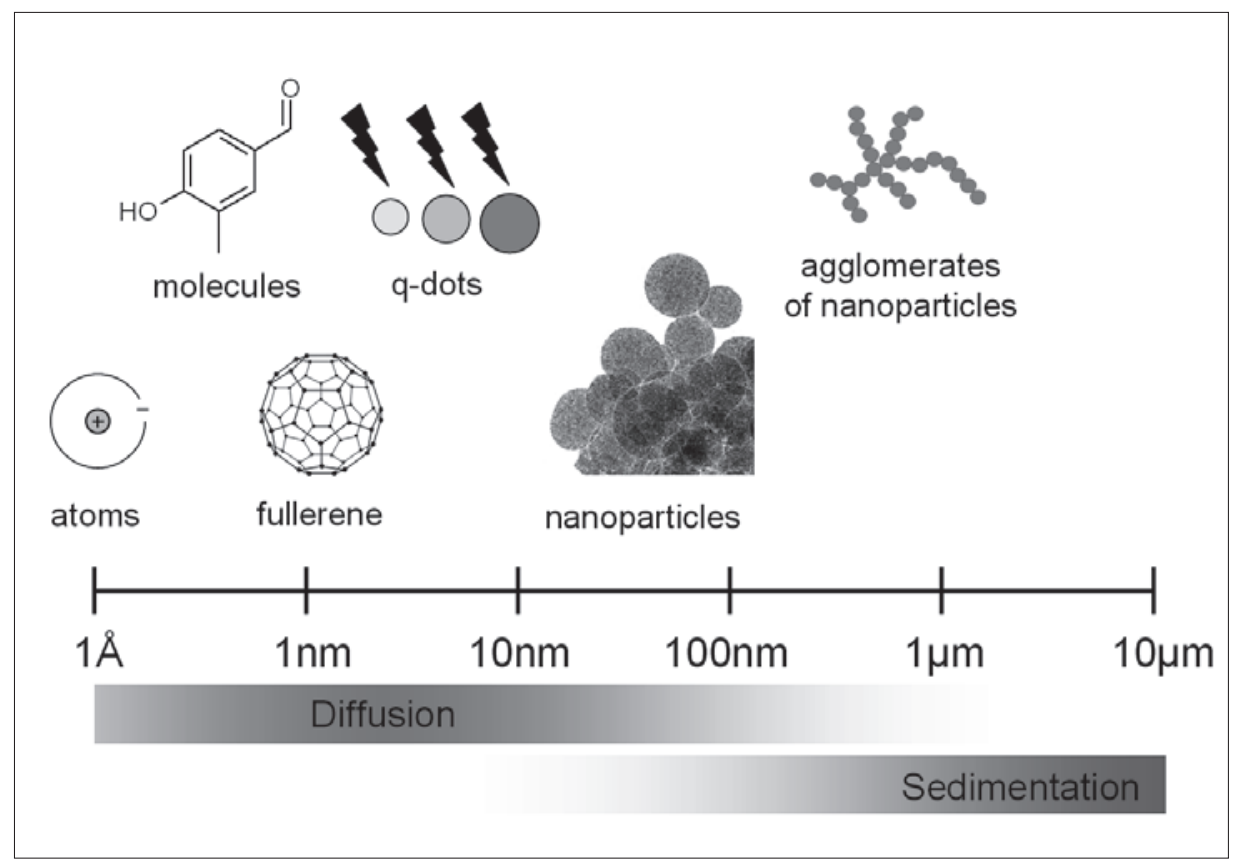

Fig. 1. Size comparison of atoms, molecules, nanomaterials, and agglomerated nanoparticles. Molecules are moved by Brownian motion, larger particles sediment or float. Particle agglomeration changes the characteristic diameter of the material, thus altering its mobility from predominantly diffusion (small, non-agglomerated) to sedimentation (larger agglomerates). glomeration rapidly alters the characteristic size of a nanomaterial. The fractal structures of agglomerated primary particles are the result of the statistical probability of one particle hitting others and occur when attractive (interparticular) forces overcome the repulsive stabilization.

\subsection{Agglomeration Results in Time- and Concentration-dependent Kinetics}

Nanomaterials have a unique transport behavior; their primary particle diameter is in the size range where diffusion is the main physical force for movement. In most applications great efforts are put into a product to make sure that particles are stable against agglomeration. However, during (accidental) uptake into an organism, or prolonged environmental exposure such stabilization typically breaks down and particles agglomerate. Once particles have formed larger groups their reduced mobility and larger mass favors sedimentation. Sedimentation and diffusion are widely known but the concept of agglomeration tends to stay within colloidal science. ${ }^{[12]}$

What are the important parameters determining the agglomeration? In order to quantitatively describe agglomeration in liquids we can use Eqn. (1) as derived from the Smoluchowski equation. ${ }^{[13]}$ This differential equation relates the change of particle number (left side of the equation) to the square of the particle number concentration (right side) and appears familiar to the physical chemist, due to its similarity with bimolecular kinetics.

$$
\frac{d n_{t}}{d t}=-\frac{4 \cdot k \cdot T}{3 \cdot \mu \cdot W} n_{t}^{2}
$$

$\mathrm{n}_{\mathrm{t}}=$ number of particles $\left[\mathrm{m}^{-3}\right]$

$\mathrm{k}=$ Boltzmann constant

$\mathrm{T}=$ temperature $[\mathrm{K}]$

$\mu=$ viscosity $[\mathrm{Pa} \mathrm{s}]$

$\mathrm{W}=$ Fuchs stability ratio, $\mathrm{W}$

The rate of agglomeration linearly scales with the number concentration squared and temperature, while it is slowed down by viscosity. In order to illustrate this rapid process better Fig. 2 compares the agglomeration kinetics of ceria nano- 


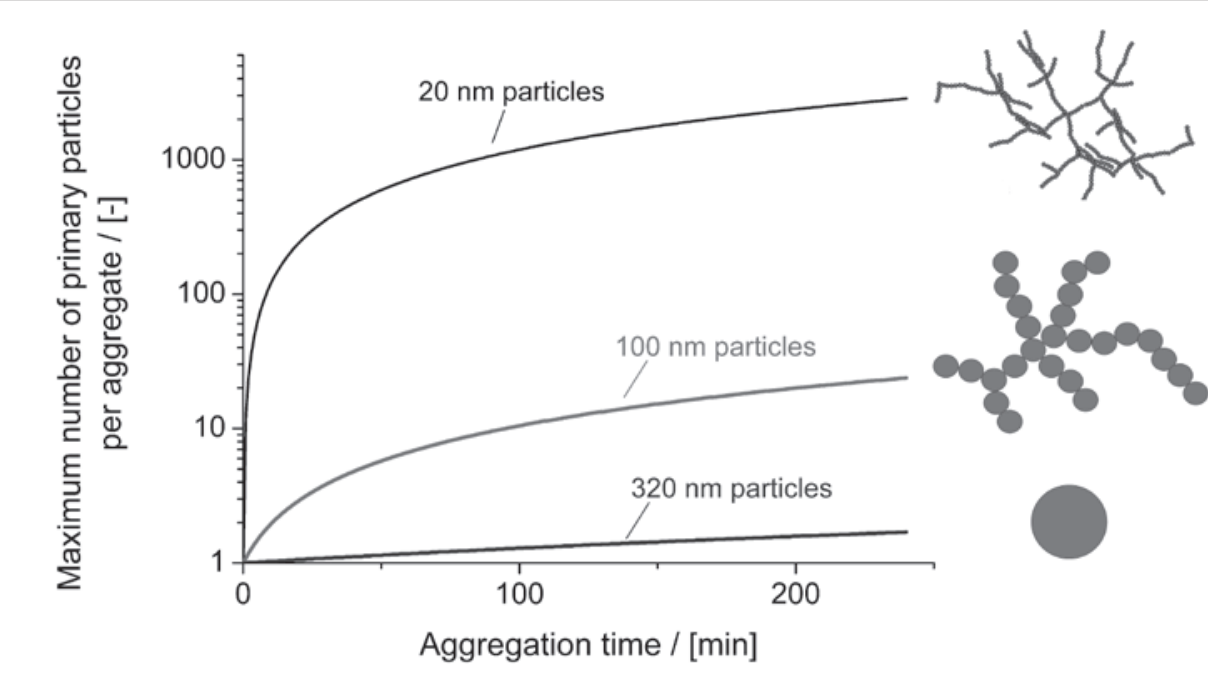

Fig. 2. Agglomeration of ceria nanoparticle suspensions with different primary particle size. The mass concentration was kept constant at 1 ppm (1 milligram per liter) in water. At these conditions diffusion limits agglomeration $(W=1)$. At the beginning, the number concentration of $20 \mathrm{~nm}$ nanoparticles is much higher when compared to larger particles. Rapid agglomeration of the small particles assembles them in bigger clusters. Therefore, agglomeration alters the mobility of the otherwise rapidly diffusing $20 \mathrm{~nm}$ particles.

particle suspensions of different sizes at a constant mass concentration of $1 \mathrm{ppm}(\mu \mathrm{g} /$ $\mathrm{ml}$ ). The smallest nanoparticles (diameter $=20 \mathrm{~nm}$ ) agglomerate strongly within seconds; an average aggregate consists of over 1000 primary particles after the duration of a typical biological experiment $(2 \mathrm{~h})$. Larger particles (diameter $=320$ nanometers) slowly stick together. This may best be explained by considering that the number concentration $\left(\# / \mathrm{m}^{3}\right)$ of smaller nanoparticles is much higher than in the case of larger particles for the same mass concentration $\left(\mathrm{kg} / \mathrm{m}^{3}\right)$. What is the consequence of this? Therefore, the 'size' of nanoparticles is a highly dynamic property, because agglomeration drastically changes in time.

Besides the number concentration of particles, agglomeration is also influenced by the temperature and viscosity of the fluid. Additionally the Fuchs stability ratio $\mathrm{W}$ is a dimensionless factor which describes the particle-particle interaction. ${ }^{[14]}$ $\mathrm{W}=1$ implies diffusion-limited aggregation, the fastest possible aggregation. Agglomeration is slowed down when the Fuchs ratio increases. As indicated, particle-particle interactions can rely mostly on electrostatic repulsion or steric hindrance. Unfortunately, there is no direct measurement for this dimensionless factor, but it can be estimated from the dynamics of the size distribution over time. Because of analytical limitations these measurements are limited to certain model compounds, mostly monodisperse particles in well-defined surroundings. ${ }^{[14,15]}$

For charge-stabilized nanomaterials we may use an experimentally easily accessible parameter to assist understanding and measurement of agglomeration processes. The so-called zeta-potential measures the charge distribution around the surface of nanomaterials and contributes to the Fuchs stability ratio W. Therefore, zeta-potential measurements can enable a qualitative comparison between different nanomaterials, as long as the steric hindrance is similar. ${ }^{[16]}$ A quantitative determination of $\mathrm{W}$ depending on the zeta-potential has been proposed by Wilson and Crimp using a computational method to predict the dispersion stability based on particle size and zeta-potential. ${ }^{[17]}$

The particle-particle interactions strongly depend on the surrounding media. Surface-charge measurements, as the zeta-potential, are highly sensitive to Adapted from Limbach et al. ${ }^{[13]}$ changes in $\mathrm{pH}$, as an alteration of $\mathrm{pH}$ not only indicates a change in acidity but also indicates a change in charge distribution within water $\left(\mathrm{H}^{+} / \mathrm{OH}^{-}\right)$and the particle surface. Increased ionic strength destabilizes the charge repulsion and favors agglomeration at higher ion concentrations, e.g. in biological fluids.

\subsection{Protein Adsorption and lons Affect Nanoparticle Mobility}

Biological fluids consist of salts and numerous biomolecules that drastically change the dynamics of nanoparticles. [18] In most cases agglomeration is strongly favored in biological media, e.g. cell culture media, because of the increased ionic strength. This effect can sometimes be compensated by the adsorption of biomolecules on the surface of nanoparticles. ${ }^{[19]}$ This adsorption process is not fully understood and is under intense investigation. ${ }^{[20]}$ Rezwan et al. showed that protein adsorption strongly effects the zeta-potential of different metal oxides. ${ }^{[21]}$ Fig. 3 shows that metal oxide nanoparticles have individual zeta-potential values in pure water (black points). The zeta-potential changes drastically in cell culture media, a mixture consisting of proteins, amino acids, salts and buffer compounds (Fig. 3, white points). Proteins adsorb on the surface and shift the surface charge to a similar value between -15 and $-20 \mathrm{mV}$. It can be assumed that steric effects of adsorbed proteins will similarly affect most inorganic nanoparticles, independent of their chemical composition. Therefore, it can be assumed that the agglomeration behavior of these particles is similar at comparable primary particle concentration, if contacted with biological fluids. This finding greatly facilitates ex-

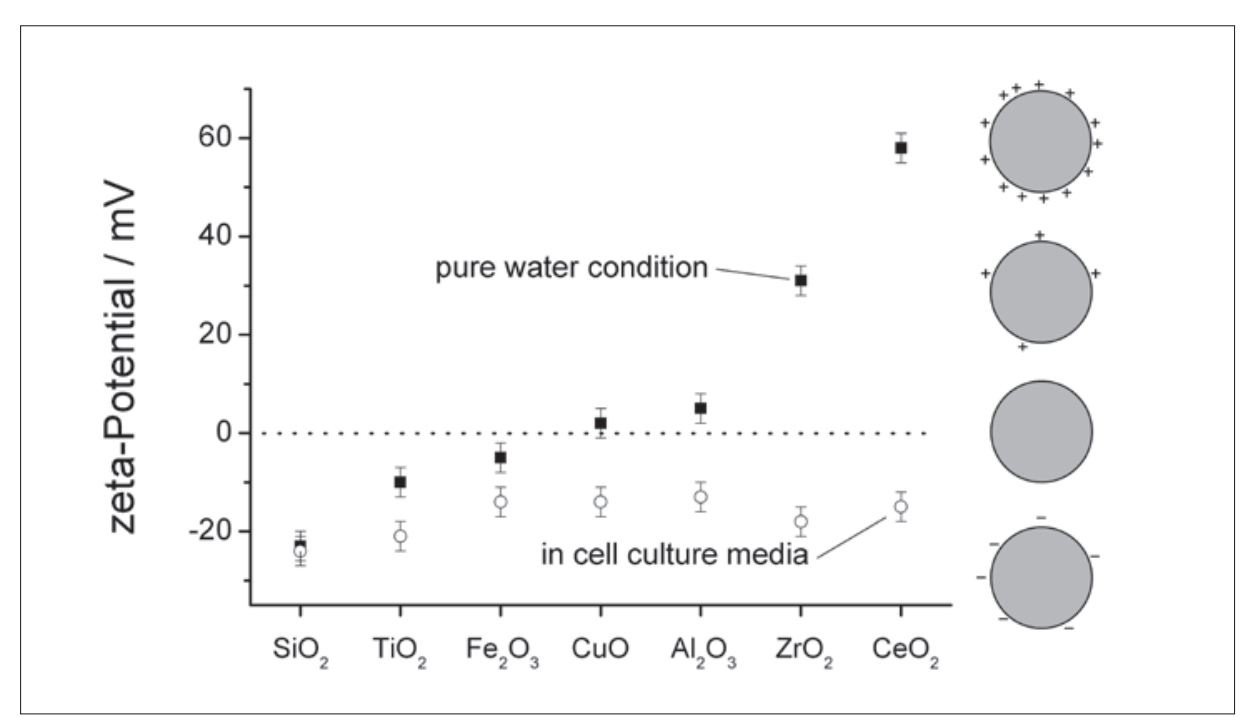

Fig. 3. The stability of different oxide nanoparticles against agglomeration is influenced by the zeta-potential. In ultra-pure water (black points), the zeta-potential of metal oxide nanoparticles shows individually characteristic values. Suspensions in cell culture medium undergo protein adsorption, which results in a similar zeta-potential and comparable agglomeration behavior. 
perimentation and prediction of nanoparticle-derived health effects, as it allows the determination of a common biodistribution pattern.

\section{Nanoparticle-Cell Interactions}

Nanoparticles strongly interact with interfaces or, as in the case of cell membranes, they typically enter cells. Classical molecules are also taken up by cells either by active processes, e.g. endocytosis, ${ }^{[22]}$ or by passive processes, where hydrophobicity and molecular weight are the major parameters. ${ }^{[23]}$ The obvious question arising is, therefore, whether nanoparticles are taken up by similar (generally applicable) mechanisms.

\subsection{Uptake of Nanomaterials in to Cells}

The uptake of nanomaterials into cells has been investigated through several analytical techniques, which indicate that uptake depends on particle size, ${ }^{[13,24,25]}$ surface charge, ${ }^{[26,27]}$ shape, ${ }^{[24,28]}$ and, obviously, on exposition time and concentration of the nanomaterial. None of these properties sounds particularly biological. Are physical material properties determining the uptake of nanomaterials in cells? It appears unlikely that cells are varying their uptake mechanism along all these parameters. Things appear clearer when taking into account that the uptake is a sequential problem: Transport of the nanomaterials to the cells (a) followed by their uptake (b). This type of problem is similar to the kinetics of heterogeneous chemical reactions and has found detailed mathematical treatment in chemical engineering. A reaction occurring between two different phases (e.g. solid particles and a liquid, or gas bubbles in a liquid) can either be limited by the transport of the reactants to the reactive site or by the reaction rate itself. The textbook way to look at such problems is to look for the ratedetermining step, the bottle-neck, in order to understand the problem. Hence, what is controlling the uptake of nanomaterials into cells, the biological uptake or the physical transport? The transport of nanomaterials to cells can be modeled by diffusion, sedimentation and agglomeration. ${ }^{[13]}$ This has been confirmed quantitatively by experimentally measuring the uptake of size-fractionated ceria nanoparticles into human lung fibroblasts over four orders of magnitude, from $100 \mathrm{ppb}$ to $100 \mathrm{ppm}$ particle exposure $(0.1$ to $100 \mathrm{mg}$ particles per liter). This study demonstrated that the transport of nanoparticles to cells is actually the limiting step of nanoparticle uptake when using classical in vitro toxicity testing. This finding does not explain how or why particles enter a cell, but explains that the measured raw data are limited by the slower of the two sequential steps, the transport. This transport is strongly influenced by the physical properties size, surface charge, exposure concentration and time and the shape of the nanomaterials. The uptake mechanism itself is not well understood at the present time.

The cellular membrane of eukaryotic cells is an evolutionary grown protective barrier against foreign particles and most molecules. Yet cells have an active transport mechanism to ensure the entry of nutrition, called endocytosis. Geiser et al. have shown that lung macrophages take up both microparticles $(1 \mu \mathrm{m})$ and nanoparticles (78 and $200 \mathrm{~nm}$ ). ${ }^{[29]}$ This is to be expected, since particle clearance is the main task of macrophages in the lung. However, microparticles were no longer taken up after a pretreatment of the cells with cytochalasin $\mathrm{D}$; a cell poison which inhibits the actin-based uptake mechanism, such as phagocytosis, but nanoparticles still entered the cells in spite of the cytochalasin D. ${ }^{[29]}$ This finding was further confirmed by the uptake of different nanoparticles in non-phagocytotic cells, for example human red blood cells ${ }^{[30]}$ and human T cells. ${ }^{[31]}$ The authors propose an unspecific mechanism (not biological, but physical) based on interfacial effects, such as electrostatic and steric interactions. ${ }^{[29]}$

Nanoparticles were found within cells, either free in the cytoplasma or enclosed by a membrane (see Fig. 4). ${ }^{[24,32]} \mathrm{A}$ recent study by Verma et al. shows the dependence of surface coatings on the uptake of gold nanoparticles into cells. ${ }^{[33]}$ Hydrophilic particles were enclosed by a membrane during the uptake into cells, whereas partially hydrophobic particles were found without any surrounding membrane in the cells. ${ }^{[33,34]}$ From this it is clear that nanoparticle uptake in cells differs from that of classical molecules.

\subsection{Acute Toxic Effects of Nanomaterials in Cells after Uptake}

The rapid uptake of nanomaterials into cells underlines the need to investigate how nanoparticles act inside cells. Toxicological assays, initially developed for chemicals, have long since been used for nanoparticles in vitro to measure acute toxic effects. ${ }^{[10]}$ In order to analyze the effects of ingested nanomaterials, studies have followed the influence of single parameters, such as size, ${ }^{[35]}$ shape, chemical composition, ${ }^{[35]}$ or crystallinity. The toxic response on physical parameters is difficult to rank, since varying physical parameters also influence the uptake (see Section 3.1.). ${ }^{[36]}$ An increased toxic response can be attributed either to a higher toxicity or an increased uptake of the material. Therefore, the toxic response should be evaluated by normalizing it with the uptake of nanomaterials and their state of agglomeration. Variation in chemical parameters such as crystallinity and composition can be more easily compared when the particle size distribution and the agglomeration behavior are similar.

\subsubsection{Solubility Strongly Influences Acute Toxicity}

Systematic investigations require changes of a single parameter only. We have found that flame spray synthesis offers the possibility to synthesize nanoparticles with a changing chemical composition but similar size distribution and shape. The agglomeration behavior is then expected to be similar, due to protein adsorption of nanoparticles (as discussed in Section 2.2.). This approach was used by Brunner et al. who investigated six industrially important metal oxide nanoparticles and compared them to asbestos or silica using appropriate cell lines. ${ }^{[37]}$ This sample set gives the possibility to measure the direct toxic response due to the similar transport properties, which is the rate-determining step of the uptake process. Insoluble nanoparticles of ceria, titania and zirconia showed no measurable toxic response for human mesothelioma cells after six days at concentrations up to $30 \mathrm{ppm}(\mu \mathrm{g} / \mathrm{ml})$. Slightly soluble nanoparticles of iron oxide and zinc oxide were toxic at similar concentrations after three days. ${ }^{[37]}$ This solubility was also proposed in other studies as a key property determining acute toxicity. ${ }^{[38,39]}$ But how can soluble nanoparticles be more toxic than metal ions at comparable concentrations?

The cellular membrane of human cells is a barrier for salts and large molecules. Nanoparticles are taken up by cells and later observed in lysosomes. In this compartment an acidic $\mathrm{pH}$ of 5.5 is present and normally assists degradation of biomolecules or, in the case of such inorganic compounds, it allows the rapid dissolution of the nanoparticles to yield considerable amounts of dissolved heavy metal ions. These toxic ions are now inside the cell and have crossed an otherwise efficient protection mechanism. The ions now damage the cell by increased osmotic pressure, reactive oxygen species formation, inactivation of enzymes and complexation, amongst others. ${ }^{[38,40]}$ The effect of smuggling ions into the cells has been described as a "nanoTrojan horse' mechanism borrowed from the Greek mythology (Fig. 4). ${ }^{[41]}$

\subsubsection{Active Catalysis Inside a Cell}

Due to their size, nanoparticles have a high specific surface and a higher percentage of their atoms are located at the surface. ${ }^{[8]}$ Therefore, surface processes gain unprecedented importance. Industrial catalysis has used these properties for over a century and nanoparticles are increasingly 
used to optimize catalysts. ${ }^{[42]}$ In toxicology some of the most damaging species in biology are oxygen-containing radicals. Industrially they are generated on purpose, through catalytically active materials, by reducing molecular oxygen. The same family of radicals has been summarized in toxicology by the term reactive oxygen species (ROS). ${ }^{[9]}$ Several studies have shown that nanoparticles can increase the number of radicals within cells in a dosedependent manner. ${ }^{[43,44]}$ The radical generation can be the result of a reduction (e.g. exposure of reduced iron particles to a cell generates reduction equivalents) or a catalytic process. This is of particular concern as catalytically active nanoparticles may generate radicals over and over again, until they are degraded or removed from the body or ecosystem. As a consequence such particles are particularly dangerous in the long term and typically escape experimental studies which usually have a (typical) focus of a few days.

\section{Possible Long-term Effects of Nanomaterials}

In contrast to acute toxic effects that can be detected within hours or days; long term effects of particle exposure will epidemiologically not be discovered for years. This has highly problematic consequences as we know from asbestos or silicosis. The biggest challenge is the difficulty to experimentally prove long-term effects prior to their (epidemiological) appearance or, ideally, before release or even invention of such a substance. The knowledge on long term effects is important at an early development stage for economic (high cost drop outs at a late stage in an industrial development pipeline) and safety reasons. In several cases it has not been possible to recognize toxic materials early enough. Asbestos was known as a non acute toxic material until it was recognized that the non-degrading fibers generate inflammation and cancer in the lung. Dichlorodiphenyltrichloroethane (DDT) was used as a powerful insecticide and known for its low toxicity to mammalians. Later on it was found that DDT was not degraded and had been accumulated in the food chain where it widely affected animals and eventually humans. Chlorofluorocarbons (CFC) have been used because of their low toxicity and their ideal properties for refrigerators. They have literally changed our food storage and transport behavior. Yet these persistent gases have also destroyed part of the ozone layer in the atmosphere.

\subsection{What Can We Learn from Asbestos, DDT and CFCs?}

In all three cases the proposed materials had new and astonishing material prop- erties and were not found to be (acutely) toxic or environmentally problematic. Unfortunately, these materials all accumulated at a certain compartment of the body (asbestos in the pleura) or ecosystem (atmosphere; food chain) which was previously not predictable. As a result their release has caused tremendous harm and damage over time. Asbestos fibers accumulated in the lungs after inhalation and can neither be cleared by macrophages nor degraded. The fibers generated inflammation in the lung and led to mesothelioma cancer. DDT was removed from plants through irrigation and was transported by the hydrological cycle into aquatic ecosystems and accumulated within the food chain. CFCs entered the stratosphere and destroyed the ozone layer, the shield layer against solar UV-radiation. Again, in all three cases these materials had unique and exciting material properties; Asbestos as an insulator for buildings, DTT as insecticide and CFCs as refrigerants. The common denominator of these materials is an unwanted accumulation due to their persistence leading to long-term health effects. Nanomaterials have new and unique material properties and their possible risks are mainly discussed in terms of their (acute) toxicity. This is short sighted. It would be most beneficial to combine the knowledge gained from asbestos, DDT, and CFCs to develop 'safe nanotechnology'.

\subsection{Safe Nanotechnology - A Proactive Approach}

A proactive approach to classify risks of nanomaterials includes both current toxicological assays to quantify acute toxic effects and a classification according to the potential for long-term effects. Therefore, currently existing toxicological protocols can be used for nanomaterials with short residence times within the body or the ecosystem. These degradable nanomaterials can be checked for their toxic response and the response of their degradation products. For persistent nanomaterials current toxicological assays inherently fail to predict potentially adverse long-term effects. New toxicity parameters have to be introduced, combining the mobility of the particles with their often unusual chemical properties and their new distribution within the body or the environment.

\subsubsection{Persistence}

The persistence of a material determines its mean dwell time in an organism or ecosystem. This inherently defines whether a material interacts shortly (acute) or for a longer time with its environment or matrix. The uptake of persistent nanoparticles in humans can, in principle, be considered as an undesired introduction of the material into the organism. We can, therefore, use an existing regulatory framework as a starting point. Current guidelines for medical implants make clear material distinctions based on the dwell time of an alien substance (e.g. an implant). This is also directly related to the definitions of pharmaceuticals versus medical implants when considering regulatory issues. For nanomaterials we have proposed a first distinction ${ }^{[45]}$ based on a material's degradation properties (similar to the presently used ISO 10993) resulting in a classification as metabolizable/degradable and persistent materials. This offers an estimate on the dwell time of the material.

Persistent nanoparticles remain within an ecosystem or an organism for a prolonged time. Most of the fundamental properties and interactions are still poorly known. Therefore, this group requires the highest degree of attention, security measures and additional investigations. Particles of this group must be considered as potentially hazardous and should be handled with special care.

\subsubsection{Degradable Nanoparticles}

In many aspects rapidly degrading nanoparticles can be regarded as similar to chemicals. This class of materials only briefly interacts with an organism or the environment. The materials are typically dissolved within a short time and degradation products can be evaluated with presently used methods. Analogous to this is the secondary metabolite analysis in pharmaceutical research. The degradation products must be followed and investigated carefully, and local accumulation of specific substances must be considered. This is important when we go back to the 'Trojan horse'-type effects where nanoparticles are efficient carriers for otherwise well-shielded materials. On an organism level the mobility of nanoparticles may help otherwise non-toxic materials to enter normally isolated areas. This can become important when checking for developmental toxicity. For degradable materials the risk evaluation can be oriented largely along the presently used material-based methods, as long as pharmacokinetics is taken into account.

\section{Conclusions}

The colorful variety of nanoparticles offers numerous attractive opportunities to our present industry and enthusiastically written technical papers provide the motivation for a rapid implementation of such novel materials into industrial products. The giant market potential of nanotechnology has mainly affected nanoparticlerelated products as a first generation of products for predominantly high tech- 


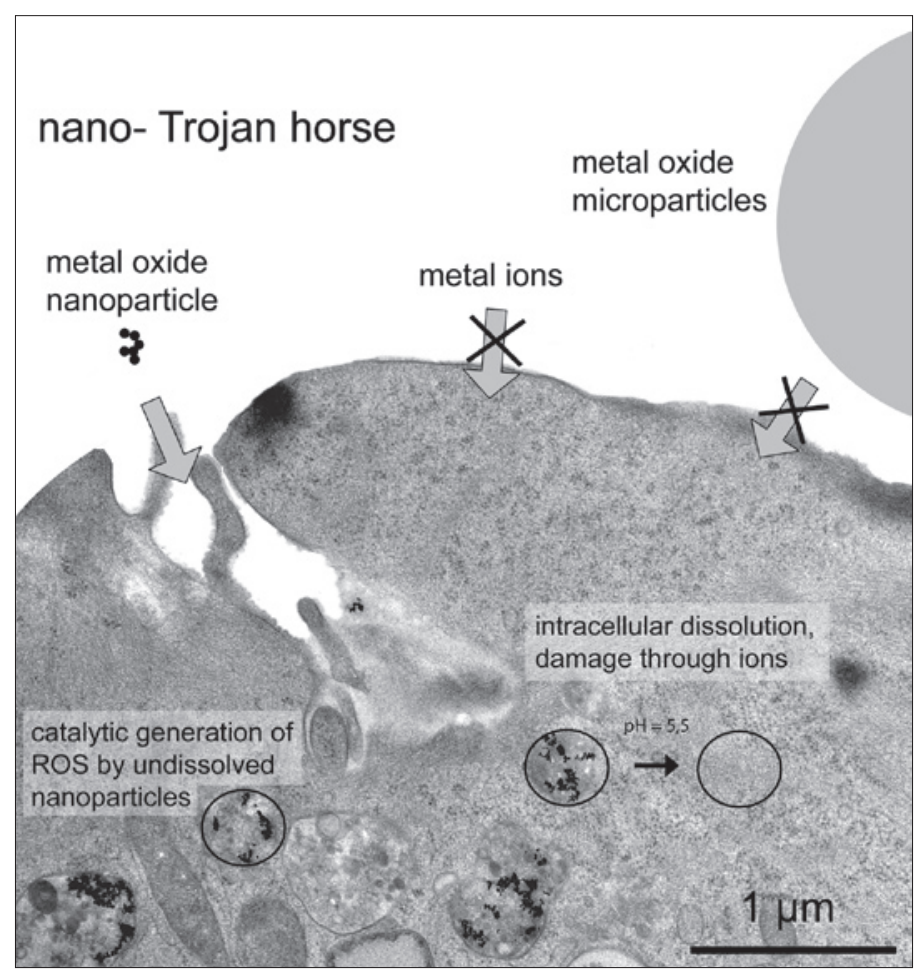

Figure 4. Nanoparticles can act as Trojan horses smuggling ions into non-phagocytotic cells. The image shows a section of a human lung fibroblast after exposure to ceria nanoparticles. Particles (black dots, arrow) are found in vesicles and transported through large sections of the cell. Specific heavy metal oxide particles can be dissolved within lysosomes (lower pH). This results in an otherwise rare transport of heavy metal ions into the cytosol.

oriented clients. Unfortunately, numerous products currently under investigation are rather persistent and literature shows that many of them are intended for large commodity use. This is not a responsible and sustainable development, as we still lack the understanding to deal with persistent nanoparticles on a technical scale or in consumer products. The distribution of persistent materials in the environment, or even within single organisms and humans, will have long-term effects on health or ecosystems. Manufacturers should therefore focus on less persistent alternatives. An early implementation of risk factors in product development will identify less persistent nanoparticles still exhibiting the desired benefits. If such a choice can be made, costly down-stream corrections should be avoided at all efforts.

\section{Acknowledgments}

Financial support by the Swiss Federal Office of Public Health (BAG, decision number 05.001872) is kindly acknowledged.

\section{Received: December 29,2008}

[1] G. Binnig, H. Rohrer, Helv. Phys. Acta 1982 55,726 .

[2] K. Donaldson, V. Stone, C. L. Tran, W. Kreyling, P. J. A. Borm, Occup. Environ. Med. 2004, 61, 727.

[3] R. F. Service, Science 2004, 304, 1732

[4] B. Brunekreef, S. T. Holgate, Lancet 2002, 360, 1233.

[5] A. Seaton, W. Macnee, K. Donaldson, D. Godden, Lancet 1995, 345, 176.

[6] A. Peters, H. E. Wichmann, T. Tuch, J. Heinrich, J. Heyder, Am. J. Respir. Crit. Care Med. 1997, 155,1376
[7] C. Sioutas, P. Koutrakis, Aerosol Sci. Technol. 1996, $25,424$.

[8] G. Oberdorster, E. Oberdorster, J. Oberdorster, Environ. Health Perspect. 2005, 113, 823. 311,622

[10] N. Lewinski, V. Colvin, R. Drezek, Small 2008, 4, 26.

[11] M. C. Powell, M. P. A. Griffin, S. Tai, Environ. Manage. 2008, 42,426

[12] S. Stoll, J. Buffle, Chimia 1995, 49, 300

[13] L. K. Limbach, Y. C. Li, R. N. Grass, T. J. Brunner, M. A. Hintermann, M. Muller, D. Gunther, W. J. Stark, Environ. Sci. Technol. 2005, 39, 9370 .

[14] M. Lattuada, H. Wu, P. Sandkuhler, J. Sefcik, M. Morbidelli, Chem. Eng. Sci. 2004, 59, 1783.

[15] N. B. Saleh, L. D. Pfefferle, M. Elimelech, Environ. Sci. Technol. 2008, 42, 7963.

[16] L. K. Limbach, R. Bereiter, E. Mueller, R. Krebs, R. Gaelli, W. J. Stark, Environ. Sci. Technol. 2008, 42, 5828. 2836.

[18] A. Petri-Fink, B. Steitz, A. Finka, J. Salaklang, H. Hofmann, Eur. J. Pharm. Biopharm. 2008, $68,129$.

[19] I. Lynch, K. A. Dawson, Nano Today 2008, 3, 40.

[20] M. Lundqvist, J. Stigler, G. Elia, I. Lynch, T. Cedervall, K. A. Dawson, Proc. Natl. Acad. Sci.

[21] K. Rezwan, A. R. Studart, J. Voros, L. J. Gauckler, J. Phys. Chem. B 2005, 109, 14469.

[22] J. L. Goldstein, R. G. W. Anderson, M. S. Brown, Nature 1979, 279, 679.

[23] B. E. Goodman, Adv. Physiol. Educ. 2002, 26, 146.

[24] B. D. Chithrani, A. A. Ghazani, W. C. W. Chan, Nano Lett. 2006, 6, 662

[25] M. J. D. Clift, B. Rothen-Rutishauser, D. M. Brown, R. Duffin, K. Donaldson, L. Proudfoot, K. Guy, V. Stone, Toxicol. Appl. Pharmacol. 2008, 232, 418.

[26] C. Wilhelm, C. Billotey, J. Roger, J. N. Pons, J. C. Bacri, F. Gazeau, Biomaterials 2003, 24 , 1001
[9] A. Nel, T. Xia, L. Madler, N. Li, Science 2006,

[17] B. A. Wilson, M. J. Crimp, Langmuir 1993, 9, U. S. A. 2008, 105, 14265
[27] D. D. Guo, C. H. Wu, X. M. Li, H. Jiang, X M. Wang, B. A. Chen, J. Nanosci. Nanotechnol. 2008, $8,2301$.

[28] A. J. Nan, X. Bai, S. J. Son, S. B. Lee, H. Ghandehari, Nano Lett. 2008, 8, 2150.

[29] M. Geiser, B. Rothen-Rutishauser, N. Kapp, S. Schurch, W. Kreyling, H. Schulz, M. Semmler, V. Im Hof, J. Heyder, P. Gehr, Environ. Health Perspect. 2005, 113, 1555.

[30] B. M. Rothen-Rutishauser, S. Schurch, B. Haenni, N. Kapp, P. Gehr, Environ. Sci. Technol. 2006, 40, 4353 .

[31] D. L. J. Thorek, A. Tsourkas, Biomaterials 2008, 29, 3583.

[32] P. Nativo, I. A. Prior, M. Brust, ACS Nano 2008 $2,1639$.

[33] A. Verma, O. Uzun, Y. H. Hu, Y. Hu, H. S. Han, N. Watson, S. L. Chen, D. J. Irvine, F. Stellacci, Nat. Mater. 2008, 7, 588 .

[34] T. Xia, L. Rome, A. Nel, Nat. Mater. 2008, 7 , 519.

[35] S. M. Hussain, K. L. Hess, J. M. Gearhart, K. T. Geiss, J. J. Schlager, 'In vitro toxicity of nanoparticles in BRL 3A rat liver cells', 13th International Workshop on In Vitro Toxicology, 2004, Zegrze, Poland, Pergamon-Elsevier Science Ltd; 2004, p. 975

[36] P. Wick, P. Manser, L. K. Limbach, U. DettlaffWeglikowska, F. Krumeich, S. Roth, W. J. Stark, A. Bruinink, Toxicol. Lett. 2007, 168, 121.

[37] T. J. Brunner, P. Wick, P. Manser, P. Spohn, R. N. Grass, L. K. Limbach, A. Bruinink, W. J. Stark, Environ. Sci. Technol. 2006, 40, 4374.

[38] N. M. Franklin, N. J. Rogers, S. C. Apte, G. E. Batley, G. E. Gadd, P. S. Casey, Environ. Sci. Technol. 2007, 41, 8484.

[39] T. Xia, M. Kovochich, M. Liong, L. Madler, B. Gilbert, H. B. Shi, J. I. Yeh, J. I. Zink, A. E. Nel, ACS Nano 2008, 2, 2121.

[40] H. L. Karlsson, P. Cronholm, J. Gustafsson, L. Moller, Chem. Res. Toxicol. 2008, 21, 1726.

[41] L. K. Limbach, P. Wick, P. Manser, R. N. Grass, A. Bruinink, W. J. Stark, Environ. Sci. Technol. 2007, 41, 4158.

[42] A. T. Bell, Science 2003, 299, 1688.

[43] C. M. Sayes, A. M. Gobin, K. D. Ausman, J. Mendez, J. L. West, V. L. Colvin, Biomaterials 2005, 26, 7587.

[44] T. C. Long, N. Saleh, R. D. Tilton, G. V. Lowry, B. Veronesi, Environ. Sci. Technol. 2006, 40, 4346.

[45] C. Meili, M. Widmer, F. Husmann, P. Gehr, F. Blank, M. Riediker, K. Schmid, L. K. Limbach, W. J. Stark, 'Synthetische Nanomaterialien: Risikobeurteilung und Risikomanagement. Grundlagenbericht zum Aktionsplan', UmweltWissen Nr. 0721. 2007, pp 256-259. 